\title{
On superintegrable symmetry-breaking potentials in $N$-dimensional Euclidean space
}

\author{
E. G. Kalnins and G. C. Williams \\ Department of Mathematics and Statistics, \\ University of Waikato, \\ Hamilton, New Zealand. \\ W. Miller, Jr. \\ School of Mathematics, University of Minnesota, \\ Minneapolis, Minnesota, 55455, U.S.A. \\ and G. S. Pogosyan* \\ Centro de Ciencias Físicas, Universidad Nacional Autónoma de México, \\ Apartado Postal 48-3, 62251 Cuernavaca, Morelos, México
}

February 28, 2002

\begin{abstract}
We give a graphical prescription for obtaining and characterising all separable coordinates for which the Schrödinger equation admits separable solutions for one of the superintegrable potentials

$$
V=\frac{1}{2} \sum_{\ell=1}^{n}\left[\frac{k_{\ell}^{2}-\frac{1}{4}}{x_{\ell}^{2}}+\omega^{2} x_{\ell}^{2}\right]+2 \omega^{2} x_{n+1}^{2}
$$

or

$$
V=-\frac{1}{2}\left(\frac{2 \alpha}{\sqrt{x_{1}^{2}+\ldots+x_{n+1}^{2}}}+\sum_{\ell=1}^{n} \frac{\frac{1}{4}-k_{\ell}^{2}}{x_{\ell}^{2}}\right) .
$$

Here $x_{n+1}$ is a distinguished cartesian variable. The algebra of second order symmetries of the resulting Schrödinger equation is given and, for the first potential,

*Permanent address: Laboratory of Theoretical Physics, Joint Institute for Nuclear Research, Dubna, Moscow Region, 141980, Russia and International Center for Advanced Studies, Yerevan State University, A.Manougian 1, 375049, Yerevan, Armenia
\end{abstract}


the closure relations of the corresponding quadratic algebra. These potentials are particularly interesting because they occur in all dimensions $n \geq 1$, the separation of variables problem is highly nontrivial for them, and many other potentials are limiting cases.

\section{Introduction}

In previous articles we have looked at two- and three-dimensional superintegrable potentials for which the Schrödinger equation is maximally superintegrable [1, $2,3,4]$. (As the first investigation of superintegrable potentials we also refer to papers [5, 6, 7]. Many examples of the relation between symmetry and variable separation are given in [8].) In this article we extend our study to the case of $N$ dimensional Euclidean space, where the requirement is that the potentials admit $2 N-1$ functionally independent second-order symmetries. As shown previously for two- and three-dimensional Euclidean space, the potentials we consider have bound state solutions which can be found in polynomial form. Using the graphical calculus developed for separable coordinates in Euclidean $N$-space and on the $n$-sphere $[9,10]$ we are able to describe systemmatically coordinate separation and bound state solutions for a large class of superintegrable systems. The basic equation that we investigate is of course Schrödinger's equation $(N=n+1)$

$$
H \Psi=-\frac{1}{2} \Delta_{N} \Psi+V(\vec{x}) \Psi=-\frac{1}{2} \sum_{\ell=1}^{N} \partial_{x_{\ell}}^{2} \Psi+V(\vec{x}) \Psi=E \Psi
$$

Our goal is to find, for two particularly chosen superintegrable potentials, all solutions of this equation via a separation of variables ansatz $\Psi=\Pi_{j=1}^{n} \psi_{j}\left(u_{j}\right)$ for all possible coordinate systems $u_{j}$. These potentials are exceptional. Not only are they superintegrable (admitting $2 N-1$ functionally independent second order constants of the motion), but they occur for all $N=n+1 \geq 2$, and contain many other special potentials as limiting cases. They have a distinguished cartesian coordinate $x_{n+1}$, and due to this symmetry breaking, these potentials do not separate in all coordinate systems for which the zero potential Schrödinger equation separates. However they do separate in a large subclass of such systems, large in the sense that the number of separable systems grows without bound as $N \rightarrow \infty$. Though the analysis is complicated, we can use the graphical characterization of all separable systems for the free equation in Euclidean $N$-space, $[9,10]$, to determine exactly which systems also separate for the given potentials. The two cases are the nonisotropic oscillator potential

$$
\text { (I) } \quad V=\frac{1}{2} \sum_{\ell=1}^{n}\left[\frac{k_{\ell}^{2}-\frac{1}{4}}{x_{\ell}^{2}}+\omega^{2} x_{\ell}^{2}\right]+2 \omega^{2} x_{n+1}^{2}+\rho x_{n+1}
$$


and the nonisotropic Coulomb potential

$$
V=-\frac{1}{2}\left(\frac{2 \alpha}{\sqrt{x_{1}^{2}+\ldots .+x_{n+1}^{2}}}+\sum_{\ell=1}^{n} \frac{\frac{1}{4}-k_{\ell}^{2}}{x_{\ell}^{2}}\right) .
$$

A third superintegrable potential, an extension of the isotropic oscillator,

$$
(I I I) \quad V=\frac{1}{2} \omega^{2}\left(x_{1}^{2}+\cdots x_{n+1}^{2}\right)+\sum_{\ell=1}^{n+1} \frac{k_{\ell}^{2}-\frac{1}{4}}{x_{\ell}^{2}}
$$

also belongs in this group. However, the variable separation problem for that case is relatively simple and is well known, [11, 12, 13]. (Ellipsoidal coordinates and all possible limiting cases of these coordinates.) Potentials (I) and (III) are nondegenerate in the sense of [3], i.e., they form an $N+1$-parameter family such that at any regular point $\mathbf{x}$ one can choose the values of the $N+1$ quantities $\partial V / \partial x_{j}$, and $\partial^{2} V / \partial x_{N}^{2}$ arbitrarily. (This is so even though for a fixed choice of parameters we can always translate coordinates in (I) so that $\rho=0$.) On the other hand, potential (II) is degenerate, it depends on only $N$ parameters.

To shed some insight on the concept of nondegeneracy we digress and examine the relationship between the potential and the invariants in a classical superintegrable system. Let the Hamiltonian be

$$
H=\sum_{i=1}^{N} p_{i}^{2}+V(\mathbf{x})
$$

where $\mathbf{x}=\left(x_{1}, \cdots, x_{N}\right)$ are cartesian coordinates. Suppose $H$ defines a superintegrable system in the sense of this paper, i.e., suppose there exist $2 N-1$ functionally independent second-order invariants $A^{(1)}=H, A^{(2)}, \cdots, A^{2 N-1)}$ :

$$
\left\{H, A^{(m)}\right\}=0, \quad m=1, \cdots, 2 N-1,
$$

where

$$
A^{(m)}=\sum_{j, k=1}^{N} A_{j k}^{(m)}(\mathbf{x}) p_{j} p_{k}+W^{(m)}(\mathbf{x}), \quad A_{j k}^{(m)}=A_{k j}^{(m)} .
$$

The integrability conditions for the matrix components and the potential are

$$
\partial_{i} A_{j k}^{(m)}+\partial_{j} A_{k i}^{(m)}+\partial_{k} A_{i j}^{(m)}=0
$$

and

$$
\begin{gathered}
A_{j k}^{(m)}\left(\partial_{j j} V-\partial_{k k} V\right)+\left(A_{k k}^{(m)}-A_{j j}^{(m)}\right) \partial_{j k} V+\sum_{\ell \neq j, k}\left(A_{k \ell}^{(m)} \partial_{j \ell} V-A_{j \ell}^{(m)} \partial_{k \ell} V\right) \\
=\sum_{i=1}^{N}\left(\partial_{k} A_{j i}^{(m)}-\partial_{j} A_{k i}^{(m)}\right) \partial_{i} V
\end{gathered}
$$


for $m=1, \cdots 2 N-1$ and $1 \leq j<k \leq N$. Expressions (5) are the equations for second-order Killing tensors [14]. The space of solutions is $N(N+1)^{2}(N+2) / 12$ dimensional and each component $A_{j k}^{(m)}$ is a second-order polynomial in the cartesian coordinates. For fixed $(j, k)$ and $m=2 \cdots, 2 N-1$, expressions (6) constitute $2 N-2$ equations for the $2 N-2$ unknowns $\partial_{j j} V-\partial_{k k} V, \partial_{j k} V, \partial_{j \ell} V, \partial_{k \ell} V$, $\ell \neq j, k$.

In all, expressions (6) constitute $M=N(N-1)^{2}$ equations for $P=(N+$ 2) $(N-1) / 2$ unknowns (the independent second derivatives of $V$ ). We can write this system in the form $\mathcal{A} \mathcal{V}_{2}=\mathcal{V}_{1}$ where $\mathcal{A}$ is $M \times P, \mathcal{V}_{2}$ is the $P \times 1$ vector of second-order derivatives of $V$, and $\mathcal{V}_{1}$ is the $M \times 1$ vector containing the terms that are first-order derivatives of $V$. We say that this system is nondegenerate provided three conditions are satisfied:

1. $\mathcal{A}$ has rank $P$, the maximum possible.

2. The augmented matrix $\mathcal{A}^{\prime}=\left(\mathcal{A}, \mathcal{V}_{1}\right)$ also has rank $P$.

These two conditions imply that we can (uniquely) solve for the $P$ secondorder derivatives $\partial_{j j} V-\partial_{N N} V, 1 \leq j<N$ and $\partial_{i k} V, 1 \leq i<k \leq N$ as linear combinations of the $N$ functions $\partial_{i} V$ with coefficients that are rational functions of the cartesian coordinates.

$$
\begin{aligned}
\partial_{j j} V-\partial_{N N} V & =\sum_{\ell=1}^{N} B_{\ell}^{j j}(\mathbf{x}) \partial_{\ell} V \\
\partial_{i k} V & =\sum_{\ell=1}^{N} B_{\ell}^{i k}(\mathbf{x}) \partial_{\ell} V
\end{aligned}
$$

(This is most easily seen, for example, if all $P$-rowed minors in $\mathcal{A}$ are nonzero. Then one can find solutions of the form (7), and if $\mathcal{A}^{\prime}$ also has rank $P$, these solutions are consistent.) From these expressions we can solve for all the third-order derivatives $\partial_{i j k} V$ and all higher-order derivatives, as linear combinations of the derivatives $\partial_{N N} V, \partial_{i} V, 1 \leq i \leq N$. We require that this process puts no further restrictions on the components.

3. All higher derivatives of $V$ can be determined unambiguously from relations (7), i.e., the integrability conditions are satisfied identically.

We say that $\mathbf{x}_{0}$ is a regular point if all the rational functions $B_{\ell}^{j j}(\mathbf{x}), B_{\ell}^{i k}(\mathbf{x})$ are well defined at $\mathbf{x}=\mathbf{x}_{0}$.

From the definition of nondegeneracy one can show that the potential $V$ is nondegenerate if and only if it is uniquely determined by the $N+2$ parameters $\partial_{N N} V\left(\mathbf{x}_{0}\right), \partial_{i} V\left(\mathbf{x}_{0}\right), V\left(\mathbf{x}_{0}\right)$, and these parameters can be prescribed arbitrarily at the regular point $\mathbf{x}_{0}$. 
All known nondegenerate superintegrable systems have the property that there is a quadratic algebra of constants of the motion. In particular there is a basis for these constants that closes under a finite number of commutators. We verify that this is indeed the case for system (I). The structure of the quadratic algebra, which we work out, provides important information for interbasis expansions relating the separable systems. On the other hand, the basis constants for the degenerate system (II) do not close under a finite number of commutators.

\section{Nonisotropic $N$-dimensional oscillator}

The first potential that we consider is

$$
V(\vec{x}, X)=\frac{1}{2} \sum_{\ell=1}^{n}\left[\frac{k_{\ell}^{2}-\frac{1}{4}}{x_{\ell}^{2}}+\omega^{2} x_{\ell}^{2}\right]+2 \omega^{2} X^{2}
$$

where $\left(x_{1}, \cdots, x_{n}, x_{n+1}\right)=(\vec{x}, X)$. From the known separable systems on the $n$-dimensional sphere and in $N$-dimensional Euclidean space $[9,10]$, we will see that the separation of variables problem can be solved for this multiparameter potential [13]. To completely describe the separable coordinates for the corresponding Schrödinger equation it will be convenient to consider first some special classes of coordinate systems. These will provide the groundwork for the complete classification of all such systems. Each such coordinate system gives rise to separation constants which are second order symmetries of the corresponding Schrödinger equation. A basis for the vector space of such symmetries for is given by

$$
\begin{gathered}
M_{i}=\partial_{x_{i}}^{2}-\omega^{2} x_{i}^{2}+\frac{\frac{1}{4}-k_{i}^{2}}{x_{i}^{2}} \\
L_{i j}=\left(x_{i} \partial_{x_{j}}-x_{j} \partial_{x_{i}}\right)^{2}+\frac{x_{i}^{2}}{x_{j}^{2}}\left(\frac{1}{4}-k_{j}^{2}\right)+\frac{x_{j}^{2}}{x_{i}^{2}}\left(\frac{1}{4}-k_{i}^{2}\right)-\frac{1}{2} \\
L=\partial_{X}^{2}-4 \omega^{2} X^{2} \\
U_{i}=\frac{1}{2}\left\{\partial_{X}, x_{i} \partial_{X}-X \partial_{x_{i}}\right\}+\omega^{2} X x_{i}^{2}+\frac{X}{x_{i}^{2}}\left(\frac{1}{4}-k_{i}^{2}\right)
\end{gathered}
$$

[Note: Strictly speaking, there should be a term $\rho X$ added to the expression (8) for the nonisotropic oscillator potential, but we can translate the $X$ coordinate 
so that this term becomes zero. (Addition of a constant to the potential is ignored.) It is worth a few words to explain why this is the case and why it doesn't occur for other superintegrable systems. In our classification of nondegenerate superintegrable systems we categorize the space of 2nd order symmetry operators for each system up to equivalence under Euclidean transformations, NOT the corresponding potentials. Once the space of symmetries is fixed, we calculate all potentials that are compatible with this space. Ordinarily each of our spaces of symmetry operators on a list of superintegrable potentials admits no proper subgroup of $E(N, R)$ as a symmetry group, i.e., any Euclidean transformation maps the space into a distinct space of symmetries. However, for case (I) the space of symmetries is invariant under translations in $X$. (The individual symmetry operators change but the space is invariant.) Thus the $\rho X$ term should appear, since it is compatible with the symmetry space, but it can be made zero by a translation. ]

We first consider separable coordinate systems for which $X$ remains unchanged. In addition we consider the various forms of coordinates for which there is a polar coordinate $r$. Typically such coordinates have the form

$$
\left(x_{1}, \ldots, x_{n}, X\right)=(r \vec{s}, X)=\left(r s_{1}, \ldots, r s_{n}, X\right)
$$

where

$$
\vec{s} \cdot \vec{s}=1
$$

For coordinates of this type the corresponding separable solutions of the Schrödinger equation have the form $\Psi(r, \vec{s}, X)=F(r) \Phi(\vec{s}) G(X)$. The effect of this choice is to reduce the problem to the equivalent one on the $(n-1)$-dimensional sphere with corresponding Rosokhatius [15] potential

$$
V_{1}=\frac{1}{2} \sum_{\ell=1}^{n} \frac{k_{\ell}^{2}-\frac{1}{4}}{s_{\ell}^{2}}
$$

Indeed the corresponding Schrödinger equation

$$
H_{1} \Phi=-\frac{1}{2} \Delta_{L B}^{(n-1)} \Phi+V_{1} \Phi=E \Phi
$$

where

$$
\Delta_{L B}^{(n-1)}=\sum_{n \geq j>k \geq 1}\left(s_{j} \partial_{s_{k}}-s_{k} \partial_{s_{j}}\right)^{2}
$$

is separable in all the coordinates for which the zero potential Schrödinger equation is separable. (The potential is a Stäckel multiplier [16].) The complete solution of this latter problem is known [17] (see also [13] for hyperspherical coordinates). The various coordinates can be constructed from a knowledge of the 
solution to this problem for elliptic coordinates in $p$-dimensions. In graphical notation, $[9,10]$, this corresponds to

$$
\left[e_{1}|\ldots| e_{p+1}\right] .
$$

The associated coordinates on the $p$ dimensional sphere have the form

$$
s_{i}^{2}=\frac{\prod_{j=1}^{p}\left(u_{j}-e_{i}\right)}{\prod_{k \neq i}\left(e_{i}-e_{k}\right)}, \quad i=1,2, \ldots, p+1
$$

where $e_{1}<u_{1}<e_{2}<\ldots<u_{p}<e_{p+1}$. In terms of these coordinates, the corresponding Schrödinger equation (16) is

$$
\begin{array}{r}
\sum_{i=1}^{p} \frac{4}{\Pi_{\ell \neq i}\left(u_{i}-u_{\ell}\right)}\left\{\Pi_{q=1}^{p+1}\left(u_{i}-e_{q}\right)\left[\partial_{u_{i}}^{2}+\frac{1}{2} \sum_{m=1}^{p+1} \frac{1}{u_{i}-e_{m}} \partial_{u_{i}}\right]\right. \\
\left.+\sum_{q=1}^{p+1}\left(\frac{1}{4}-k_{q}^{2}\right) \frac{\Pi_{k \neq q}\left(e_{q}-e_{k}\right)}{\prod_{j=1}^{p}\left(u_{j}-e_{q}\right)}\right\} \Psi=-2 E \Psi .
\end{array}
$$

The separation equations are

$$
\begin{array}{r}
4\left\{\Pi_{q=1}^{p+1}\left(u_{i}-e_{q}\right)\left[\partial_{u_{i}}^{2}+\frac{1}{2} \sum_{m=1}^{p+1} \frac{1}{u_{i}-e_{m}} \partial_{u_{i}}\right]\right\} \psi_{i}\left(u_{i}\right) \\
+\left[\sum_{\ell=1}^{p+1}\left(\frac{1}{4}-k_{\ell}^{2}\right) \frac{\Pi_{j \neq \ell}\left(u_{i}-e_{j}\right)}{\left(u_{i}-e_{\ell}\right)}-2 E u_{i}^{p-1}+\sum_{\ell=0}^{p-2}(-1)^{\ell} \lambda_{\ell}^{p} u_{i}^{\ell}\right] \psi_{i}\left(u_{i}\right)=0
\end{array}
$$

for $i=1, \cdots, p$. We find the solutions of this equation as follows. If we make the ansatz

$$
\Psi=\left(\prod_{\ell=1}^{p+1} s_{\ell}^{k_{\ell}+1 / 2}\right) \prod_{m=1}^{q}\left(\sum_{j=1}^{p+1} \frac{s_{j}^{2}}{\theta_{m}-e_{j}}\right)
$$

the zeros must satisfy [17]

$$
\sum_{j=1}^{p+1} \frac{k_{j}+1}{\theta_{q}-e_{j}}+\sum_{l \neq q} \frac{2}{\theta_{q}-\theta_{l}}=0 .
$$

This ansatz is based on the identity

$$
\sum_{j=1}^{p+1} \frac{s_{j}^{2}}{\theta-e_{j}}=-\frac{\Pi_{i=1}^{p}\left(u_{i}-\theta\right)}{\Pi_{j=1}^{p+1}\left(\theta-e_{j}\right)}
$$

The operators that specify the separation constants are

$$
\left.\Lambda_{j}^{p}=\sum_{i_{p} \neq i_{p+1}} S_{i_{1} \ldots i_{p-1}}^{j+1}\left(L_{i_{p} i_{p+1}}-k_{i_{p}}^{2}-k_{i_{p+1}}^{2}\right)+\sum_{i_{p+1}}(j+1) S_{i_{1} \ldots i_{p}}\left(k_{i_{p+1}}^{2}-\frac{1}{4}\right)\right] .
$$


The eigenvalue of $E$ and these operators are

$$
\begin{aligned}
E & =\frac{1}{2}\left[2 q+p+\sum_{j=1}^{p+1} k_{j}\right]^{2}-\frac{1}{8}(p-1)^{2} \\
\lambda_{j}^{p} & =\sum_{i_{p+1}=1}^{p+1}\left[4 S_{i_{1} \ldots i_{p}}^{p-j} \sum_{m=1}^{q} \frac{\left(k_{i_{p+1}}+1\right)}{\theta_{m}-e_{i_{p+1}}}+2 S_{i_{1} \ldots i_{p-1}}^{p-j-1} k_{i_{p}} k_{i_{p+1}}\right. \\
& \left.+2(j+1) S_{i_{1} \ldots i_{p}}^{p-j-1} k_{i_{p+1}}+\frac{1}{4}(j+1)(2 j+5) S_{i_{1} . . i_{p+1}}^{p-1}\right] .
\end{aligned}
$$

Here the symbol $S_{j_{1} . . j_{t}}^{r}$ denotes the sum of all products of $r$ elements taken from $e_{j_{1}}, \ldots, e_{j_{t}}$, where $j_{1}, \ldots, j_{t}$ are all different and the symbol is symmetric in the indices $j_{1}, . ., j_{t}$, e.g.,

$$
S_{123}^{1}=e_{1}+e_{2}+e_{3}, \quad S_{123}^{2}=e_{1} e_{2}+e_{1} e_{3}+e_{2} e_{3} .
$$

Effectively the sum in these formulas is taken over $i_{p+1}$ as indicated. In order to obtain the basic building blocks we need to illustrate how coordinates corresponding to the diagram

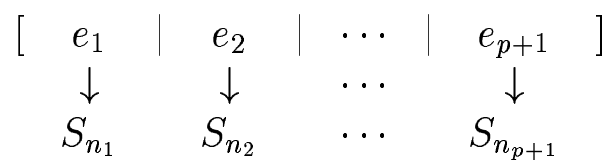

give rise to separable solutions and how the wave functions can be computed. A convenient choice of coordinates in this case is

$$
\left(v_{1}, \ldots, v_{N}\right)=\left(s_{1} \vec{w}_{n_{1}}, \ldots, s_{p+1} \vec{w}_{n_{p+1}}\right),
$$

where $\vec{w}_{n_{j}}=\left(w_{n_{j}}^{1}, . ., w_{n_{j}}^{n_{j}+1}\right)$ is a unit vector on the sphere $S_{n_{j}}$ and $N=\sum_{j=1}^{p+1} n_{j}+$ $p+1$. If we now redefine the $k_{i}^{\prime} s$ according to

$$
\vec{k}=\left(k_{1}, \ldots, k_{N}\right)=\left(K_{11}, \ldots, K_{1 n_{1}+1}, K_{21}, \ldots, K_{2 n_{2}+1}, \ldots, K_{p+1 n_{p+1}+1}\right)
$$

then a solution of the Schrödinger equation can be obtained in the form

$$
\Psi=\prod_{\ell=1}^{p+1} s_{\ell}^{\Gamma_{\ell}-n_{\ell}+1 / 2}\left[\prod_{m=1}^{s}\left\{\left(\sum_{j=1}^{p+1} \frac{s_{j}^{2}}{\theta_{m}-e_{j}}\right) \chi_{n_{m}}\left(w_{n_{m}}\right)\right\}\right]
$$

where

$$
\Gamma_{\ell}=2 m_{\ell}+n_{\ell}+1+\sum_{h=1}^{n_{\ell}+1} K_{\ell h}, \quad E=\frac{1}{2}\left(2 s+\sum_{\ell=1}^{N} \Gamma_{\ell}+p\right)^{2}-\frac{1}{8}(p-1)^{2}
$$


and

$$
\left[\Delta_{L B}^{\left(n_{\ell}\right)}+\sum_{h=1}^{n_{\ell}+1} \frac{\frac{1}{4}-K_{\ell h}^{2}}{\left(w_{n_{\ell}}^{h}\right)^{2}}\right] \chi_{n_{\ell}}\left(\vec{w}_{n_{m}}\right)=\left[\frac{1}{4}\left(n_{\ell}-1\right)^{2}-\Gamma_{\ell}^{2}\right] \chi_{n_{\ell}}\left(\vec{w}_{n_{m}}\right) .
$$

The equation satisfied by the zeros $\theta_{m}$ is

$$
\sum_{n=1}^{p+1} \frac{\Gamma_{n}+1}{\theta_{s}-e_{n}}+\sum_{r \neq s} \frac{2}{\theta_{s}-\theta_{r}}=0 .
$$

The separation equations are

$$
\begin{gathered}
4 \prod_{q=1}^{p+1}\left(u_{i}-e_{q}\right)\left[\partial_{u_{i}}^{2}+\frac{1}{2} \sum_{m=1}^{p+1} \frac{n_{m}}{u_{i}-e_{m}} \partial_{u_{i}}\right] \psi_{i}\left(u_{i}\right)+ \\
\left\{\sum_{\ell=1}^{p+1}\left[\frac{1}{4}\left(n_{\ell}-1\right)^{2}-\Gamma_{\ell}^{2}\right] \frac{\Pi_{j \neq \ell}\left(u_{i}-e_{j}\right)}{\left(u_{i}-e_{\ell}\right)}-2 E u_{i}^{p-1}+\sum_{\ell=1}^{p-1}(-1)^{\ell} \lambda_{\ell} u_{i}^{\ell-1}\right\} \psi_{i}\left(u_{i}\right)=0 .
\end{gathered}
$$

The eigenvalues $\lambda_{\ell}$ are given by the formulas used for general elliptic coordinates corresponding to the diagram

$$
\left[e_{1}|\ldots| e_{p+1}\right]
$$

but with the replacement $k_{j} \rightarrow \Gamma_{j}, j=1, . ., p+1$. This enables us to give all the separable coordinates of the type for the potential under consideration.

We give an example of how this works. Consider the coordinate system described by the diagram



This diagram corresponds to the choice of coordinates

$$
\left(s_{1}, s_{2}, s_{3}, s_{4}, s_{5}, s_{6}\right)=\left(t_{1} w_{1}, t_{1} w_{2}, t_{1} w_{3}, t_{2}, t_{3} r_{1}, t_{3} r_{2}\right)
$$

where

$$
\begin{aligned}
t_{i}^{2}=\frac{\Pi_{j=1}^{2}\left(u_{j}-e_{i}\right)}{\Pi_{k \neq i}\left(e_{i}-e_{k}\right)}, & i=1,2,3 \\
w_{i}^{2}=\frac{\Pi_{j=1}^{2}\left(v_{j}-a_{i}\right)}{\Pi_{k \neq i}\left(a_{i}-a_{k}\right)}, & i=1,2,3
\end{aligned}
$$




$$
r_{i}^{2}=\frac{y-c_{i}}{\prod_{k \neq i}\left(c_{i}-c_{k}\right)}, \quad i=1,2
$$

The solutions to the corresponding Schrödinger equation with potential

$$
V=\frac{1}{2} \sum_{\ell=1}^{6} \frac{k_{\ell}^{2}-\frac{1}{4}}{s_{\ell}^{2}}
$$

have the form $\Psi=\Psi_{1}\left(u_{1}, u_{2}\right) \Psi_{2}\left(v_{1}, v_{2}\right) \Psi_{3}(y)$ where

$$
\begin{gathered}
\Psi_{3}(y)=r_{1}^{k_{5}+1 / 2} r_{2}^{k_{6}+1 / 2} \Pi_{\ell=1}^{m}\left(\frac{r_{1}^{2}}{\varphi_{\ell}-c_{1}}+\frac{r_{2}^{2}}{\varphi_{\ell}-c_{2}}\right), \\
\Psi_{2}\left(v_{1}, v_{2}\right)=w_{1}^{k_{1}+1 / 2} w_{2}^{k_{2}+1 / 2} w_{3}^{k_{3}+1 / 2} \Pi_{b=1}^{n}\left(\frac{w_{1}^{2}}{\theta_{b}-a_{1}}+\frac{w_{2}^{2}}{\theta_{b}-a_{2}}+\frac{w_{3}^{2}}{\theta_{b}-a_{3}}\right), \\
\Psi_{1}\left(u_{1}, u_{2}\right)=t_{1}^{\Gamma_{1}-1 / 2} t_{2}^{\Gamma_{2}+1 / 2} t_{3}^{\Gamma_{3}} \Pi_{j=1}^{o}\left(\frac{t_{1}^{2}}{\mu_{j}-e_{1}}+\frac{t_{2}^{2}}{\mu_{j}-e_{2}}+\frac{t_{3}^{2}}{\mu_{j}-e_{3}}\right),
\end{gathered}
$$

with $\Gamma_{1}=2 n+2+k_{1}+k_{2}+k_{3}, \Gamma_{2}=k_{4}$ and $\Gamma_{3}=2 m+1+k_{5}+k_{6}$. The equations satisfied by the zeros are

$$
\begin{gathered}
\frac{k_{5}+1}{\varphi_{s}-c_{1}}+\frac{k_{6}+1}{\varphi_{s}-c_{2}}+\sum_{r \neq s} \frac{2}{\varphi_{s}-\varphi_{r}}=0, \\
\frac{k_{1}+1}{\theta_{s}-a_{1}}+\frac{k_{2}+1}{\theta_{s}-a_{2}}+\frac{k_{3}+1}{\theta_{s}-a_{3}}+\sum_{r \neq s} \frac{2}{\theta_{s}-\theta_{r}}=0, \\
\frac{\Gamma_{1}+1}{\mu_{s}-e_{1}}+\frac{\Gamma_{2}+1}{\mu_{s}-e_{2}}+\frac{\Gamma_{3}+1}{\mu_{s}-e_{3}}+\sum_{r \neq s} \frac{2}{\mu_{s}-\mu_{r}}=0 .
\end{gathered}
$$

The separation equations for these solutions are

$$
\begin{array}{r}
4\left(y-c_{1}\right)\left(y-c_{2}\right)\left\{\partial_{y}^{2}+\frac{1}{2}\left[\frac{1}{y-c_{1}}+\frac{1}{y-c_{2}}\right] \partial_{y}\right] \Psi_{3}(y)+ \\
{\left[\left(c_{1}-c_{2}\right)\left(\frac{\frac{1}{4}-k_{5}^{2}}{y-c_{1}}-\frac{\frac{1}{4}-k_{6}^{2}}{y-c_{2}}\right)+\Gamma_{3}^{2}\right] \Psi_{3}(y)=0}
\end{array}
$$




$$
\begin{aligned}
&\left\{4\left(v_{i}-a_{1}\right)\left(v_{i}-a_{2}\right)\left(v_{i}-a_{3}\right)\left[\partial_{v_{i}}^{2}+\frac{1}{2}\left(\frac{1}{v_{i}-a_{1}}+\frac{1}{v_{i}-a_{2}}+\frac{1}{v_{i}-a_{3}}\right) \partial_{v_{i}}\right]+\right. \\
&\left(\frac{1}{4}-k_{1}^{2}\right) \frac{\left(v_{i}-a_{2}\right)\left(v_{i}-a_{3}\right)}{\left(v_{i}-a_{1}\right)}+\left(\frac{1}{4}-k_{2}^{2}\right) \frac{\left(v_{i}-a_{1}\right)\left(v_{i}-a_{3}\right)}{\left(v_{i}-a_{2}\right)}+ \\
&\left.\left.\left(\frac{1}{4}-k_{3}^{2}\right) \frac{\left(v_{i}-a_{2}\right)\left(v_{i}-a_{1}\right)}{\left(v_{i}-a_{3}\right)}\right)-2\left(\frac{1}{4}-\Gamma_{1}^{2}\right) v_{i}+\lambda\right\} \psi_{2}^{i}\left(v_{i}\right)=0
\end{aligned}
$$

where $\Psi_{2}\left(v_{1}, v_{2}\right)=\psi_{2}^{1}\left(v_{1}\right) \psi_{2}^{2}\left(v_{2}\right)$,

$$
\begin{gathered}
\left\{4\left(u_{i}-e_{1}\right)\left(u_{i}-e_{2}\right)\left(u_{i}-e_{3}\right)\left[\partial_{u_{i}}^{2}+\frac{1}{2}\left(\frac{3}{u_{i}-e_{1}}+\frac{1}{u_{i}-e_{2}}+\frac{2}{u_{i}-e_{3}}\right) \partial_{u_{i}}\right]+\right. \\
\left(1-\Gamma_{1}^{2}\right) \frac{\left(u_{i}-e_{2}\right)\left(u_{i}-e_{3}\right)}{\left(u_{i}-e_{1}\right)}+\left(\frac{1}{4}-\Gamma_{2}^{2}\right) \frac{\left(u_{i}-e_{1}\right)\left(u_{i}-e_{3}\right)}{\left(u_{i}-e_{2}\right)}(48) \\
\left.-\Gamma_{3}^{2} \frac{\left(u_{i}-e_{2}\right)\left(u_{i}-e_{1}\right)}{\left(u_{i}-e_{3}\right)}-2 E u_{i}+\eta\right\} \varphi_{1}^{i}\left(u_{i}\right)=0
\end{gathered}
$$

where $\Phi_{1}\left(u_{1}, u_{2}\right)=\varphi_{1}^{1}\left(u_{1}\right) \varphi_{1}^{2}\left(u_{2}\right)$ and

$$
E=\left(2(m+n+o+3)+k_{1}+k_{2}+k_{3}+k_{4}+k_{5}+k_{6}\right)^{2}-\frac{9}{4} .
$$

The operators describing the separation constants $\Gamma_{3}^{2}, \lambda, \frac{1}{4}-\Gamma_{1}^{2}, \eta$ and $2 E$ are

$$
\begin{aligned}
\Gamma_{3}^{2} & =L_{56}+1-k_{5}^{2}-k_{6}^{2} \\
\lambda & =-a_{1} L_{23}-a_{2} L_{13}-a_{3} L_{12}-\frac{1}{2}\left(a_{1}+a_{2}+a_{3}\right), \\
\frac{1}{4}-\Gamma_{1}^{2} & =L_{12}+L_{13}+L_{23} \\
\eta & =e_{1}\left(L_{45}+L_{46}\right)+e_{2}\left(L_{15}+L_{25}+L_{35}+L_{16}+L_{26}+L_{36}\right) \\
& +e_{3}\left(L_{14}+L_{24}+L_{34}\right)-\frac{1}{2}\left(e_{1}+e_{2}+e_{3}\right) \\
2 E & =\sum_{1<i<j<6} L_{i j},
\end{aligned}
$$

respectively. The eigenvalues $\lambda$ and $\eta$ are given by

$$
\begin{aligned}
\lambda & =-a_{1}\left(k_{2} k_{3}+k_{2}+k_{3}\right)-a_{2}\left(k_{1} k_{3}+k_{1}+k_{3}\right)-a_{3}\left(k_{2} k_{1}+k_{2}+k_{1}\right) \\
& -\frac{3}{2}\left(a_{1}+a_{2}+a_{3}\right)+4 a_{2} a_{3}\left(k_{1}+1\right) \sum_{\ell=1}^{n} \frac{1}{\theta_{\ell}-a_{1}} \\
& +4 a_{2} a_{1}\left(k_{3}+1\right) \sum_{\ell=1}^{n} \frac{1}{\theta_{\ell}-a_{3}}+4 a_{3} a_{1}\left(k_{2}+1\right) \sum_{\ell=1}^{n} \frac{1}{\theta_{\ell}-a_{2}}
\end{aligned}
$$




$$
\begin{aligned}
\eta & =-e_{1}\left(k_{4} \Gamma_{3}+k_{4}+\Gamma_{3}\right)-e_{2}\left(\Gamma_{1} \Gamma_{3}+\Gamma_{1}+\Gamma_{3}\right) \\
& -e_{3}\left(k_{4} \Gamma_{1}+k_{4}+\Gamma_{1}\right)-\frac{3}{2}\left(\Gamma_{1}+k_{4}+\Gamma_{3}\right)+4 e_{2} e_{3}\left(\Gamma_{1}+1\right) \sum_{\ell=1}^{o} \frac{1}{\mu_{\ell}-e_{1}} \\
& +4 e_{2} e_{1}\left(\Gamma_{3}+1\right) \sum_{\ell=1}^{o} \frac{1}{\mu_{\ell}-e_{3}}+4 e_{3} e_{1}\left(k_{4}+1\right) \sum_{\ell=1}^{o} \frac{1}{\mu_{\ell}-e_{2}} .
\end{aligned}
$$

We have established the following result: For the potential $V_{1}$, solution of the

Schrödinger equation via the separation of variables ansatz is possible in all the coordinate systems for which the Helmholtz equation on the sphere is separable. In terms of the graphical calculus, all separable systems on the sphere are constructed from tree graphs whose components come from the branching rule

$$
\begin{array}{c|c|c|c}
e_{1} & e_{2} & \cdots & e_{q} \\
\downarrow & \downarrow & \cdots & \downarrow \\
S_{1} & S_{2} & \cdots & S_{q}
\end{array}
$$

where $S_{j}$ indicates coordinates on the sphere of dimension $n_{j}=\operatorname{dim} S_{j}$.

Now that we see how the separation of variables works on the sphere for the potential $V_{1}$, we can describe how separation of variables works in the case of the potential

$$
V_{2}=\frac{1}{2} \sum_{\ell=1}^{n}\left[\frac{k_{\ell}^{2}-\frac{1}{4}}{x_{\ell}^{2}}+\omega^{2} x_{\ell}^{2}\right] .
$$

The corresponding Schrödinger equation can be solved by separation of variables in all the coordinates for which the corresponding Helmholtz equation can be solved. The coordinates for which this is possible consist of a disjoint sum of graphs of the form

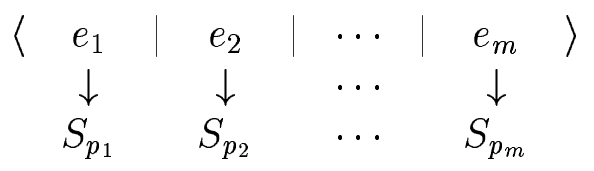

where the symbol $<e_{1}|\ldots| e_{m}>$ denotes elliptic coordinates in $m$ dimensional Euclidean space [10], viz

$$
x_{i}^{2}=\frac{\prod_{j=1}^{m}\left(z_{j}-e_{i}\right)}{\prod_{k \neq i}\left(e_{i}-e_{k}\right)}, \quad i=1 \cdots m
$$

The standard choice of coordinates for the above diagram is

$$
\vec{r}=\left(x_{1} \vec{w}_{1}, \ldots, x_{m} \vec{w}_{m}\right)
$$


where each $\vec{w}_{j}$ lies on a unit sphere of dimension $p_{j}$. The corresponding Schrödinger equation can be written in the form

$$
\left\{\partial_{\vec{x}} \cdot \partial_{\vec{x}}-\omega^{2} \vec{x} \cdot \vec{x}+\sum_{\ell=1}^{m}\left[\frac{1}{4}\left(p_{\ell}-1\right)^{2}-\Gamma_{\ell}^{2}\right] \frac{1}{x_{\ell}^{2}}+2 E\right\} \Psi=0
$$

where

$$
\Delta_{L B}^{(j)} \Psi=\left[\frac{1}{4}\left(p_{j}-1\right)^{2}-\Gamma_{j}^{2}\right] \Psi
$$

for $j=1, . ., m$. If we write this out in elliptical coordinates $z_{k}$ the equality assumes the form

$$
\begin{array}{r}
\left\{\sum _ { i = 1 } ^ { m } \left[\frac{4}{\prod_{\ell \neq i}\left(z_{i}-z_{\ell}\right)} \Pi_{q=1}^{m}\left(z_{i}-e_{q}\right)\left[\partial_{z_{i}}^{2}+\frac{1}{2} \sum_{\ell=1}^{m} \frac{p_{\ell}}{z_{i}-e_{\ell}} \partial_{z_{i}}\right]\right.\right. \\
\left.\left.+\left[\frac{1}{4}\left(p_{i}-1\right)^{2}-\Gamma_{i}^{2}\right] \frac{\Pi_{k \neq i}\left(e_{i}-e_{k}\right)}{\Pi_{q=1}^{n}\left(z_{i}-e_{q}\right)}\right]+\omega^{2} \sum_{\ell=1}^{m}\left(z_{\ell}-e_{\ell}\right)+2 E\right\} \Psi=0 .
\end{array}
$$

The separation equations are

$$
\begin{array}{r}
4 \Pi_{q=1}^{m}\left(z_{i}-e_{q}\right)\left[\partial_{z_{i}}^{2}+\frac{1}{2} \sum_{\ell=1}^{m} \frac{p_{\ell}}{z_{i}-e_{\ell}} \partial_{z_{i}}\right] \varphi_{i}\left(u_{i}\right) \\
+\left\{\sum_{k=1}^{n}\left[\frac{1}{4}\left(p_{k}-1\right)^{2}-\Gamma_{k}^{2}\right] \frac{\Pi_{j \neq k}\left(z_{i}-e_{j}\right)}{\left(z_{i}-e_{k}\right)}-\omega^{2} u^{m}\right. \\
\left.-\left(2 E+\omega^{2} \sum_{k=1}^{n} e_{k}\right) z_{i}^{m-1}+\sum_{\ell=1}^{m-2} \lambda_{\ell-1}^{m} z_{i}^{\ell}\right\} \varphi_{i}\left(u_{i}\right)=0 .
\end{array}
$$

To obtain the polynomial solutions in the usual way we look for solutions of the form

$$
\Psi=\exp \left(-\frac{\omega}{2} \sum_{j=1}^{m} x_{j}^{2}\right)\left[\Pi_{j=1}^{M} x_{j}^{\frac{1}{2}\left(1-p_{j}\right)+\Gamma_{j}}\right] \Pi_{\ell=1}^{m}\left(\sum_{s=1}^{m} \frac{x_{s}^{2}}{\theta_{\ell}-e_{s}}-1\right) .
$$

With this ansatz the $\theta_{m}$ satisfy

$$
\sum_{s=1}^{n} \frac{\Gamma_{s}+1}{\theta_{\ell}-e_{s}}+\sum_{t \neq \ell} \frac{2}{\theta_{\ell}-\theta_{t}}-\omega=0 .
$$

The energy $E$ is given by

$$
E=\omega\left(2 q+M+\sum_{j=1}^{n} \Gamma_{j}\right)
$$


The operators whose eigenvalues are the separation parameters $\lambda_{\ell-1}^{n}$ are

$$
\begin{array}{r}
\Lambda_{\ell-1}^{n}=\sum_{i_{1} \neq i_{2}} S_{i_{3}, \ldots, i_{\ell-1}}^{\ell-1}\left(L_{i_{1} i_{2}}-k_{i_{1}}^{2}-k_{i_{2}}^{2}\right) \\
+\sum_{i_{1}} S_{i_{2} \ldots i_{\ell-1}}^{\ell}\left[M_{i_{1}}+(\ell-n)\left(\frac{1}{4}-\Gamma_{i_{1}}^{2}\right)\right]-S^{\ell+1} \omega^{2} .
\end{array}
$$

The eigenvalues can be readily calculated as

$$
\begin{aligned}
\lambda_{\ell-1}^{n} & =-\sum_{i_{1} \neq i_{2}} S_{i_{3}, \ldots, i_{\ell-1}}^{\ell-1}\left(1+k_{i_{1}}+k_{i_{2}}\right)^{2}+ \\
& -\sum_{i_{1}} S_{i_{2} \ldots i_{\ell-1}}^{\ell}\left[2\left(k_{i_{1}}+1\right)\left(\omega+2 \sum_{\ell=1}^{q} \frac{1}{\theta_{\ell}-e_{i_{1}}}+(n-\ell)\left(\frac{1}{4}-\Gamma_{i_{1}}^{2}\right)\right]-S^{\ell+1} \omega^{2} .\right.
\end{aligned}
$$

For the potential $V_{2}$ exactly the coordinate systems constructed from disjoint graphs, viz

$$
\begin{array}{ccc|ccc|c|c}
\left\langle e_{1}\right. & \cdots & e_{k} & \rangle+\cdots+\langle & c_{1} & \cdots & \left.c_{s}\right\rangle \\
\downarrow & \cdots & \downarrow & \cdots & \downarrow & \cdots & \downarrow \\
S_{p_{1}} & \cdots & S_{p_{k}} & \cdots & S_{q_{1}} & \cdots & S_{q_{s}}
\end{array}
$$

enable the corresponding Schrödinger equation to be solved via separation of variables.

We are now in a position to give a complete solution to the problem of separation of variables for our original problem: the Schrödinger equation in $n+1$ dimensions with potential

$$
V=\frac{1}{2} \sum_{\ell=1}^{n}\left(\frac{k_{\ell}^{2}-\frac{1}{4}}{x_{\ell}^{2}}+\omega^{2} x_{\ell}^{2}\right)+2 \omega^{2} X^{2}
$$

There are two possible types of separable coordinate systems. The first and most obvious are the disjoint coordinate systems of the type already used for the previous potential $V_{2}$ and excluding the coordinate $X$. For a coordinate system of this type the $X$ coordinate is not connected to any other coordinate and can factored out of the solution via the ansatz $\Psi=\Psi\left(x_{1}, . ., x_{n}\right) \psi(X)$ where $\psi(X)=e^{-\omega X^{2}} H_{q}(\sqrt{2 \omega} X)$.

The only type of separable coordinate system that is linked to the coordinate $X$ can be taken as

$$
\left(x_{1}, . ., x_{n}, X\right)=\left(\xi \eta \vec{w}, \frac{1}{2}\left(\xi^{2}-\eta^{2}\right)\right)=\left(x^{\prime} \vec{w}, y^{\prime}\right)=\left(\vec{x}, y^{\prime}\right) .
$$

Here $\vec{w} \cdot \vec{w}=1$ is a vector on the $n-1$ dimensional sphere. [Note: We could also take coordinates of the form $\left(x_{1}, . ., x_{n}, X\right)=\left(\vec{x}^{\prime} w, x_{p+1}, . ., x_{n}, X\right)$ upon suitable 
rearrangement of the Cartesian coordinates $x_{1}, . ., x_{n}$. If we separated the dependence on $x_{p+1}, \ldots, x_{n}$ out we would then have essentially the coordinate system (72) but with $n=p$.] Returning to (72), if we write the Schrödinger equation for these coordinates and use our potential we have, with $\Psi=\Psi\left(x^{\prime}, y^{\prime}\right) \psi(w)$, that the equation for $\Psi\left(x^{\prime}, y^{\prime}\right)$ is

$$
\begin{gathered}
{\left[\partial_{x^{\prime}}^{2}+\frac{n}{x^{\prime}} \partial_{x^{\prime}}+\partial_{y^{\prime}}^{2}-\omega^{2}\left(x^{\prime 2}+4 y^{\prime 2}\right)+\left(\frac{1}{4}(n-1)^{2}-\Gamma_{n}^{2}\right) \frac{1}{x^{\prime 2}}+2 E\right] \Psi\left(x^{\prime}, y^{\prime}\right)=0} \\
\sum_{n \geq i>j \geq 1}\left(x_{i} \partial_{x_{j}}-x_{j} \partial_{x_{i}}\right)^{2} \psi(w)=\left[\frac{1}{4}(n-1)^{2}-\Gamma_{n}^{2}\right] \psi(w)
\end{gathered}
$$

where $\Gamma_{n}=2 q+n+\sum_{l=1}^{n} k_{l}$. This equation assumes a more transparent form if we write $\Psi\left(x^{\prime}, y^{\prime}\right)=\left(x^{\prime}\right)^{-n / 2} \Phi\left(x^{\prime}, y^{\prime}\right)$. Then is

$$
\left[\partial_{x^{\prime}}^{2}+\partial_{y^{\prime}}^{2}-\omega^{2}\left(x^{2}+4 y^{\prime 2}\right)+\left(\frac{1}{4}-\Gamma_{n}^{2}\right) \frac{1}{x^{\prime 2}}+2 E\right] \Phi\left(x^{\prime}, y^{\prime}\right)=0
$$

This is exactly the equation we obtain in the case of two dimensional space, with the exception of the occurence of $\Gamma_{n}$ [1]. If we use $x^{\prime}$ and $y^{\prime}$ as separable coordinates then the corresponding solutions are

$$
\Phi\left(x^{\prime}, y^{\prime}\right)=\left(y^{\prime}\right)^{\frac{1}{2}+\Gamma_{n}} e^{\left[-\omega\left(\frac{1}{2}\left(y^{\prime}\right)^{2}+\left(x^{\prime}\right)^{2}\right)\right]} L_{n}^{\Gamma_{n}}\left(\omega y^{\prime 2}\right) H_{q}\left(\sqrt{ } 2 \omega x^{\prime}\right) .
$$

(However, this is equivalent to the earlier type in which $X=y^{\prime}$ is isolated from the other coordinates.) Alternatively if we choose parabolic coordinates

$$
x^{\prime}=\xi \eta, \quad y^{\prime}=\frac{1}{2}\left(\xi^{2}-\eta^{2}\right)
$$

then $y^{\prime}$ is linked and the equation assumes the form

$$
\left\{\frac{1}{\xi^{2}+\eta^{2}}\left[\partial_{\xi}^{2}+\partial_{\eta}^{2}\right]-\omega^{2}\left(\xi^{4}-\xi^{2} \eta^{2}+\eta^{4}\right)+\frac{\frac{1}{4}-\Gamma_{n}^{2}}{\xi^{2} \eta^{2}}+2 E\right\} \Phi=0 .
$$

This equation admits separable solutions $\varphi_{1}(\xi) \varphi_{2}(\eta)$ which satisfy the separation equations

$$
\left(\partial_{\mu}^{2}-\omega^{2} \mu^{6}+\frac{\frac{1}{4}-\Gamma_{n}^{2}}{\mu^{2}}+2 E \mu^{2}+\epsilon \beta\right) \varphi_{j}(\mu)=0,
$$

where $\mu=\xi, \eta$ according as $j=1,2$, respectively. To obtain the bound state solutions for this problem we make use of the identity [1]

$$
\frac{x \cdot x}{\lambda^{2}}+2 y^{\prime}-\lambda^{2}=\frac{\left(\xi^{2}-\lambda^{2}\right)\left(\eta^{2}+\lambda^{2}\right)}{\lambda^{2}} .
$$


If we look for solutions of the form

$$
\Phi=\Pi_{j=1}^{q}\left(\frac{\vec{x} \cdot \vec{x}}{\lambda_{j}^{2}}+2 y^{\prime}-\lambda_{j}^{2}\right)
$$

we see that the $\lambda_{j}$ satisfy

$$
\frac{4\left(\Gamma_{n}+1\right)}{\lambda_{m}^{2}}+\sum_{\ell \neq m} \frac{4}{\lambda_{\ell}^{2}-\lambda_{m}^{2}}-2 \omega \lambda_{m}^{2}=0
$$

and the eigenvalue $\beta$ is given by the expression

$$
\beta=2\left(\Gamma_{n}+1\right) \Pi_{j=1}^{q} \lambda_{j}^{2}\left(\sum_{k=1}^{q} \lambda_{k}^{-2}\right)
$$

which is the eigenvalue of the operator $2 \sum_{\ell=1}^{n} U_{\ell}$. given by (12).

We have established that the coordinate systems for which the Schrödinger equation with potential $V$ is separable are of two types:

[1]. Coordinate systems for which the $X$ coordinate remains isolated. This corresponds to the systems which separate for $V_{2}$.

$$
\begin{array}{c|c|cccc|c|c}
\left\langle e_{1}\right. & \cdots & e_{k} & \rangle+\cdots+\langle & c_{1} & \cdots & c_{s}
\end{array}+<a>
$$

[2]. Coordinate systems which are associated with disjoint sum of graphs of the form

$$
\begin{array}{ccc|ccccc|ccc}
\left\langle e_{1}\right. & \cdots & e_{k} & \rangle+\cdots+\langle & c_{1} & \cdots & c_{s} & \rangle+ & (0) \\
\downarrow & \cdots & \downarrow & \cdots & \downarrow & \cdots & \downarrow & \downarrow \\
S_{p_{1}} & \cdots & S_{p_{k}} & \cdots & S_{q_{1}} & \cdots & S_{q_{s}} & S_{s}
\end{array}
$$

Here, (0) is the basic parabolic coordinate system.

How do we know that this potential doesn't separate in coordinate systems other than those listed above? The characterization of the separable systems by $N$ second-order symmetry operators in involution is crucial here. In [10] these operators are listed in detail for the zero-potential case in Euclidean spaces and on the sphere. We have computed the vector space of symmetry operators for our potential, and given a basis in $(9,10,11,12)$. In order that our equation separate in a given coordinate system, we must be able to construct operators from this vector space that agree in their differential terms with the characterizing operators listed in [10]. For example, the fact that there is no operator analagous to $L_{n+1, n+1}$ in $(9,10,11,12)$ means that the coordinate $x_{n+1}$ cannot be part of any separable ellipsoidal or hyperspherical coordinate system. 


\section{Nonisotropic $N$-dimensional Coulomb problem}

In addition to the potential we have considered thus far we can also discuss the potential (which is the $N$-dimensional generalization of the tree-dimensional superintegrable potential [7])

$$
V=-\frac{1}{2}\left\{\frac{2 \alpha}{\sqrt{x_{1}^{2}+\ldots+x_{n+1}^{2}}}+\frac{\frac{1}{4}-k_{1}^{2}}{x_{1}^{2}}+\ldots+\frac{\frac{1}{4}-k_{n}^{2}}{x_{n}^{2}}\right\}
$$

The corresponding Schrödinger equation

$$
\left\{\partial_{x_{1}}^{2}+\ldots+\partial_{x_{n+1}}^{2}+\frac{2 \alpha}{\sqrt{x_{1}^{2}+\ldots .+x_{n+1}^{2}}}+\frac{\frac{1}{4}-k_{1}^{2}}{x_{1}^{2}}+\ldots+\frac{\frac{1}{4}-k_{n}^{2}}{x_{n}^{2}}+2 E\right\} \Psi=0
$$

admits symmetries (here $J_{h \ell}=x_{h} \partial_{x_{\ell}}-x_{\ell} \partial_{x_{h}}$ )

$$
\begin{gathered}
L_{i j}=J_{i j}^{2}+\left(\frac{1}{4}-k_{j}^{2}\right) \frac{x_{i}^{2}}{x_{j}^{2}}+\left(\frac{1}{4}-k_{i}^{2}\right) \frac{x_{j}^{2}}{x_{i}^{2}}-\frac{1}{2} \\
L_{i, n+1}=J_{i, n+1}^{2}+\left(\frac{1}{4}-k_{i}^{2}\right) \frac{x_{n+1}^{2}}{x_{i}^{2}}-\frac{1}{2} \\
L=\frac{1}{2} \sum_{i=1}^{n}\left(\left\{\partial_{x_{i}}, J_{n+1, i}\right\}+\left(\frac{1}{4}-k_{i}^{2}\right) \frac{x_{n+1}}{x_{i}^{2}}\right)+\frac{\alpha x_{n+1}}{\sqrt{x_{1}^{2}+\ldots+x_{n+1}^{2}}} .
\end{gathered}
$$

The Schrödinger equation admits a separation of variables in coordinates

$$
\left(x_{1}, \ldots, x_{n+1}\right)=r \vec{s}
$$

where $\vec{s}=\left(s_{1}, \ldots, s_{n+1}\right)$ is expressed in terms of any separable coordinate system on the $n$ dimensional sphere. If we look for solutions of Schrödinger's equation of the form $\Psi=F(r) \Phi(\vec{s})$, the equations for $F(r)$ and $\Phi(\vec{s})$ are

$$
\begin{gathered}
\left\{\partial_{r}^{2}+\frac{n}{r} \partial_{r}+\frac{2 \alpha}{r}-\left[\Gamma^{2}-\frac{(n-1)^{2}}{4}\right] \frac{1}{r^{2}}+2 E\right\} F(r)=0 \\
\left(\Delta_{L B}^{(n)}-\sum_{i=1}^{n} \frac{k_{i}^{2}-\frac{1}{4}}{s_{i}^{2}}\right) \Phi=-\left[\Gamma^{2}-\frac{(n-1)^{2}}{4}\right] \Phi
\end{gathered}
$$


where $\Gamma=\ell+M+\frac{1}{2}, M=2 m+n-1+k_{1}+k_{2}+\ldots+k_{n}$. This equation has solutions

$$
F(r)=\exp (-\sqrt{-2 E} r) r^{\Gamma-\frac{n-1}{2}} L_{n_{r}}^{2 \Gamma}(2 \sqrt{-2 E} r)
$$

where $L_{\ell}^{\beta}(z)$ is a Laguerre polynomial and $n_{r}=0,1,2 \ldots$ is radial quantum number. The quantisation condition on the energy levels is

$$
E=-\frac{\alpha^{2}}{2\left(n_{r}+\ell+M+1\right)^{2}},
$$

The second set of coordinates in which separation of variables is possible is a version of parabolic cordinates, viz.

$$
x_{i}=\xi \eta s_{i}, \quad x_{n+1}=\frac{1}{2}\left(\xi^{2}-\eta^{2}\right), \quad i=1,2, \ldots n .
$$

In these coordinates the Schrödinger equation has the form

$$
\begin{array}{r}
\left\{\partial_{\xi}^{2}+\frac{n-1}{\xi} \partial_{\xi}+\partial_{\eta}^{2}+\frac{n-1}{\eta} \partial_{\eta}+4 \alpha+2 E\left(\xi^{2}+\eta^{2}\right)\right. \\
\left.+\left(\frac{1}{\xi^{2}}+\frac{1}{\eta^{2}}\right)\left[\Delta_{L B}^{(n-1)}-\sum_{i=1}^{n} \frac{k_{i}^{2}-\frac{1}{4}}{s_{i}^{2}}\right]\right\} \Psi=0
\end{array}
$$

With $\Psi=(\xi \eta)^{\frac{1}{2}(1-n)} \hat{\Psi}(\xi, \eta) \Phi\left(s_{1}, \ldots, s_{n}\right)$, the equations for $\hat{\Psi}$ and $\Phi$ have the form

$$
\begin{gathered}
\left\{\partial_{\xi}^{2}+\partial_{\eta}^{2}+4 \alpha-\left(\frac{1}{\xi^{2}}+\frac{1}{\eta^{2}}\right)\left(M^{2}-\frac{1}{4}\right)+2 E\left(\xi^{2}+\eta^{2}\right)\right\} \hat{\Psi}=0 \\
\left(\Delta_{L B}^{(n-1)}-\sum_{i=1}^{n} \frac{k_{i}^{2}-\frac{1}{4}}{s_{i}^{2}}\right) \Phi=-\left[M^{2}-\frac{(n-2)^{2}}{4}\right] \Phi
\end{gathered}
$$

where $M=2 q+(n-1)+k_{1}+\ldots+k_{n}$. The equation (96) is essentially that we have already looked at. The separable solutions for the wave functions $\hat{\Psi}$ in the parabolic coordinates $\xi, \eta$ is

$$
\hat{\Psi}(\xi, \eta)=\exp \left[-\sqrt{-2 E}\left(\xi^{2}+\eta^{2}\right)\right](\xi \eta)^{M+\frac{1}{2}} L_{n_{1}}^{M}(2 \sqrt{-2 E} \xi) L_{n_{2}}^{M}(2 \sqrt{-2 E} \eta)
$$

where $n_{1}, n_{2}=0,1,2, .$. and the energy spectrum have the form

$$
E=-\frac{\alpha^{2}}{2\left(n_{1}+n_{2}+M+1\right)^{2}}
$$


The equation (96) can be also solved by a separation of variables by regarding $\xi$ and $\eta$ as cartesian coordinates. We could also choose polar or elliptical coordinates and solve our problem by separation of variables. In elliptical coordinates we can obtain a solution for $\hat{\Psi}$ by writing

$$
\hat{\Psi}(\xi, \eta)=\exp \left[-\sqrt{-2 E}\left(\xi^{2}+\eta^{2}\right)\right](\xi \eta)^{\left(M+\frac{1}{2}\right)} \Pi_{j=1}^{s}\left(\frac{\xi^{2}}{\theta_{j}-e_{1}}+\frac{\eta^{2}}{\theta_{j}-e_{2}}-1\right)(
$$

where the $\theta_{j}$ satisfy

$$
\frac{M+1}{\theta_{j}-e_{1}}+\frac{M+1}{\theta_{j}-e_{2}}+\sum_{j \neq m} \frac{2}{\theta_{m}-\theta_{j}}-\sqrt{-2 E}=0
$$

and we have the quantisation condition

$$
E=-\frac{\alpha^{2}}{2(s+M+1)^{2}}
$$

We could also write this expression in terms of Cartesian coordinates by using

$$
\xi^{2}=\sqrt{x_{1}^{2}+\ldots+x_{n+1}^{2}}+x_{n+1}, \quad \eta^{2}=\sqrt{x_{1}^{2}+. .+x_{n+1}^{2}}-x_{n+1} .
$$

In conclusion, this potential separates in three classes of coordinate systems:

$$
\begin{array}{ccc}
\text { polar } & \text { ellipsoidal } & \text { parabolic } \\
<a> & <a, b> & (o) \\
\downarrow & \downarrow & \downarrow \\
S_{n} & S_{n-1} & S_{n-1}
\end{array}
$$

Here, $S_{k}$ is the sphere of dimension $k$.

Again, we can check that this potential doesn't separate in coordinate systems other than those listed above by making use of characterization of the separable systems by $N$ second-order symmetry operators in involution as given in [10]. We have computed the vector space of symmetry operators for our potential, and given a basis in $(86,87,88)$. In order that our equation separate in a given coordinate system, we must be able to construct operators from this vector space that agree in their differential terms with the characterizing operators listed in [10].

\section{Operator characterizations}

Associated with the various separations of variables are the operator characterisations of the individual systems. The operators form a quadratic algebra which determines the nature of the dynamical symmetries associated with the Schrödinger 
equation. For the first potential, we give the defining relations for the quadratic algebra generated by $M_{i}, L_{j k}, L$ and $U_{i}$. (We note that the algebra of operators for the last potential cannot be closed polynomially under repeated commutation. However if we discard $L$ closure is again possible.) Returning to our first potential, the first commutators are

$$
\begin{gathered}
{\left[M_{i}, M_{j}\right]=\left[M_{i}, L_{j k}\right]=\left[M_{i}, L\right]=\left[M_{i}, U_{j}\right]=0,} \\
{\left[M_{i}, L_{i j}\right]=Q_{i j}=Q_{[i j]}, \quad\left[L_{i j}, L_{i k}\right]=R_{i j k}=R_{[i j k]}, \quad\left[L_{i j}, L_{k l}\right]=0} \\
{\left[L_{i j}, L\right]=\left[L_{i j}, U_{k}\right]=0, \quad\left[M_{i}, U_{i}\right]=A_{i},} \\
{\left[L_{i j}, U_{i}\right]=B_{i j}=B_{[i j]}, \quad\left[L, U_{i}\right]=-A_{i}, \quad\left[U_{i}, U_{j}\right]=(-1 / 4) Q_{i j},}
\end{gathered}
$$

where the $Q_{i j}, R_{i j k}$ and $B_{i j}$ are totally antisymmetric. The commutators of the $M_{i}, L_{j k}, L, U_{k}$ and $Q_{p q}$ are

$$
\begin{gathered}
{\left[M_{i}, Q_{i j}\right]=4\left\{M_{i}, M_{j}\right\}+16 \omega^{2} L_{i j},\left[M_{i}, Q_{j k}\right]=0} \\
{\left[L_{i j}, Q_{i j}\right]=4\left\{M_{i}, L_{i j}\right\}-4\left\{M_{j}, L_{i j}\right\}+16\left(1-k_{j}^{2}\right) M_{i}-16\left(1-k_{i}^{2}\right) M_{j}} \\
{\left[L_{i j}, Q_{i k}\right]=4\left\{M_{i}, L_{j k}\right\}-4\left\{M_{j}, L_{i k}\right\}, \quad\left[U_{i}, Q_{i j}\right]=4\left\{M_{i}, U_{j}\right\}} \\
{\left[L_{i j}, Q_{k l}\right]=\left[L, Q_{i j}\right]=\left[U_{i}, Q_{j k}\right]=0 .}
\end{gathered}
$$

The commutators of $M_{i}, L_{j k}, L, U_{k}$ and with $R_{k l m}$ are

$$
\begin{gathered}
{\left[M_{i}, R_{i j k}\right]=4\left\{M_{k}, L_{i j}\right\}-4\left\{M_{j}, L_{i k}\right\},\left[M_{i}, R_{j k l}\right]=0,} \\
{\left[L_{i j}, R_{i j k}\right]=4\left\{L_{i j}, L_{j k}\right\}-4\left\{L_{i j}, L_{i k}\right\}+16\left(1-k_{i}^{2}\right) L_{j k}-16\left(1-k_{j}^{2}\right) L_{i k},} \\
{\left[L_{i j}, R_{i k l}\right]=4\left\{L_{i k}, L_{j l}\right\}-4\left\{L_{j k}, L_{i l}\right\}, \quad\left[U_{i}, R_{i j k}\right]=4\left\{L_{i j}, U_{k}\right\}-4\left\{L_{i k}, U_{j}\right\},} \\
{\left[L, R_{i j k}\right]=\left[L_{i j}, R_{k l m}\right]=\left[U_{i}, R_{j k l}\right]=0 .}
\end{gathered}
$$

The commutators of $M_{i}, L_{j k}, L, U_{k}$ and with $A_{m}$ are

$$
\begin{gathered}
{\left[M_{i}, A_{i}\right]=16 \omega^{2} U_{i},\left[M_{i}, A_{j}\right]=0,\left[L_{i j}, A_{i}\right]=4\left\{M_{i}, U_{j}\right\}-4\left\{M_{j}, U_{i}\right\}} \\
{\left[L, A_{i}\right]=-16 \omega^{2} U_{i},\left[U_{i}, A_{i}\right]=2 M_{i}^{2}-2\left\{M_{i}, L\right\}+8 \omega^{2}\left(1-k_{i}^{2}\right)} \\
{\left[L_{i j}, A_{k}\right]=0, \quad\left[U_{i}, A_{j}\right]=2 M_{i} M_{j}+4 \omega^{2} L_{i j} .}
\end{gathered}
$$

The commutators of $M_{i}, L_{j k}, L, U_{k}$ and with $B_{m n}$ are

$$
\begin{gathered}
{\left[M_{i}, B_{i j}\right]=-4\left\{M_{i}, U_{i}\right\},\left[M_{i}, B_{j k}\right]=0} \\
{\left[L_{i j}, B_{i j}\right]=-4\left\{L_{i j}, U_{i}\right\}+4\left\{L_{i j}, U_{j}\right\}-16\left(1-k_{j}^{2}\right) U_{i}+16\left(1-k_{i}^{2}\right) U_{j},} \\
{\left[L_{i j}, B_{i k}\right]=4\left\{L_{k i}, U_{j}\right\}-4\left\{L_{k j}, U_{i}\right\},\left[L_{i j}, B_{k l}\right]=0}
\end{gathered}
$$




$$
\begin{gathered}
{\left[U_{i}, B_{i j}\right]=\left\{L_{i j}, L\right\}-2\left\{L_{i j}, L\right\}-4\left\{U_{i}, U_{j}\right\}-4\left(1-k_{i}^{2}\right) M_{i},} \\
{\left[U_{i}, B_{j k}\right]=\left\{L_{i k}, M_{j}\right\}-\left\{L_{i j}, M_{k}\right\}, \quad\left[L, B_{i j}\right]=4\left\{M_{j}, U_{i}\right\}-4\left\{M_{i}, U_{j}\right\} .}
\end{gathered}
$$

The commutators of the $Q_{i j}, R_{k l m}, A_{k}$ and $B_{m n}$ amongst themselves are

$$
\begin{gathered}
{\left[Q_{i j}, Q_{i k}\right]=4\left\{M_{i}, Q_{j k}\right\},\left[Q_{i j}, Q_{k l}\right]=0,\left[Q_{i j}, R_{i j k}\right]=-4\left\{L_{i j}, Q_{i k}\right\}-4\left\{L_{i j}, Q_{j k}\right\},} \\
{\left[Q_{i j}, R_{i k l}\right]=4\left\{L_{i k}, Q_{l j}\right\}+4\left\{L_{i l}, Q_{j k}\right\},\left[Q_{i j}, R_{i k l}\right]=0,\left[Q_{i j}, A_{i}\right]=-4\left\{M_{i}, A_{j}\right\},} \\
{\left[Q_{i j}, B_{i j}\right]=-4\left\{L_{i j}, A_{i}\right\}-4\left\{L_{i j}, A_{j}\right\},\left[Q_{i j}, B_{i k}\right]=-4\left\{L_{i k}, A_{j}\right\}+4\left\{U_{i}, Q_{j k}\right\},} \\
{\left[R_{i j k}, R_{i j l}\right]=4\left\{L_{i j}, R_{i k l}\right\}-4\left\{L_{k l}, R_{i j m}\right\}-4\left\{L_{j m}, R_{i k l}\right\}+4\left\{L_{k m}, R_{i j l}\right\},} \\
{\left[R_{i j k}, R_{l m n}\right]=\left[R_{i j k}, A_{l}\right]=\left[Q_{i j}, B_{k l}\right]=0,} \\
{\left[R_{i j k}, B_{i j}\right]=4\left\{L_{k i}, B_{i j}\right\}-4\left\{L_{j k}, B_{i j}\right\}-4\left\{U_{i}, R_{i j k}\right\}+4\left\{U_{j}, R_{i j k}\right\}} \\
+16\left(1-k_{i}^{2}\right) B_{j k}-16\left(1-k_{j}^{2}\right) B_{k i}, \\
{\left[R_{i j k}, B_{i l}\right]=4\left\{L_{j l}, B_{i k}\right\}-4\left\{L_{k l}, B_{i j}\right\}-4\left\{U_{j}, R_{k l i}\right\}+4\left\{U_{k}, R_{l i j}\right\},} \\
{\left[R_{i j k}, B_{l m}\right]=0,\left[A_{i}, A_{j}\right]=4 \omega^{2} Q_{i j},} \\
{\left[A_{i}, B_{i j}\right]=\left\{M_{i}, Q_{i j}\right\}-4\left\{U_{i}, A_{j}\right\},\left[A_{i}, B_{j k}\right]=\left\{M_{i}, Q_{j k}\right\}-4 \omega^{2} R_{i j k},} \\
{\left[B_{i j}, B_{i k}\right]=4\left\{U_{i}, B_{j k}\right\}+\left\{L_{k l}, Q_{i k}\right\}-\left\{L_{i k}, Q_{i j}\right\}-16\left(1-k_{i}^{2}\right) Q_{j k},} \\
{\left[B_{i j}, B_{k l}\right]=-\left\{L_{i k}, Q_{j l}\right\}+\left\{L_{i l}, Q_{j k}\right\}+\left\{L_{j k}, Q_{i l}\right\}-\left\{L_{j l}, Q_{i k}\right\} .}
\end{gathered}
$$

All the commutators of the $M_{i}, L_{m n}, Q_{p q}$ and $R_{r s t}$ can be expressed in terms of quadratic symmetric products of themselves. The algebra therefore closes quadratically. We should note that only some of these commutators exist in dimension 5 or higher. There are relations between the symmetric products of the generators of this algebra. These are listed in the appendix.

\section{Acknowledgements}

G.S.P. acknowledges the Consejo Nacional de Ciencia y Tecnología (México) for a Cátedra Patrimonial Nivel II, and Russian Foundation for Basic Research, grant RFBR N00-02-81023. 


\section{Appendix}

Here we list identities between symmetric products of the generators of the quadratic algebra for the first potential. The list is exhaustive, except that identities having overall more indices than those which appear in each of the terms of the identity have not been listed, e.g., an identities of the form

$$
\left\{D_{i}, D_{j}\right\}+\left\{D_{j}, D_{k}\right\}+\left\{D_{k}, D_{i}\right\}=0, i \neq j \neq k
$$

Our exhaustive list is as follows. In all the formulas it is assumed that indices with different labels have distinct numerical values. Definitions of each of the terms are given in (104).

$$
\begin{aligned}
& \left\{M_{i}, R_{i j k}\right\}=\left\{L_{i j}, Q_{i k}\right\}-\left\{L_{i k}, Q_{i j}\right\}-\left\{L_{i i}, Q_{j k}\right\} \\
& \left\{M_{i}, R_{j k l}\right\}=-\left\{L_{i j}, Q_{k l}\right\}-\left\{L_{i k}, Q_{l j}\right\}-\left\{L_{i l}, Q_{j k}\right\} . \\
& Q_{i j}^{2}=(8 / 3)\left\{L_{i j}, M_{i}, M_{j}\right\}+16 \omega^{2} L_{i j}^{2}-16\left(1-k_{j}^{2}\right) M_{i}^{2} \\
& -16\left(1-k_{i}^{2}\right) M_{j}^{2}+(64 / 3)\left\{M_{i}, M_{j}\right\}-(128 / 3) \omega^{2} L_{i j}-64\left(1-k_{i}^{2}\right)\left(1-k_{j}^{2}\right), \\
& \left\{Q_{i j}, Q_{i k}\right\}=(8 / 3)\left\{L_{i j}, M_{i}, M_{k}\right\}+(8 / 3)\left\{L_{i k}, M_{i}, M_{j}\right\}-(8 / 3)\left\{L_{j k}, M_{i}, M_{i}\right\} \\
& +32 \omega^{2}\left(1-k_{i}^{2}\right)\left\{L_{i j}, L_{i k}\right\}-8\left\{M_{j}, M_{k}\right\}-32\left(1-k_{i}^{2}\right) L_{j k}, \\
& \left\{Q_{i j}, Q_{k l}\right\}=-(8 / 3)\left\{L_{i k}, M_{j}, M_{l}\right\}-(8 / 3)\left\{L_{j l}, M_{i}, M_{k}\right\} \\
& +(8 / 3)\left\{L_{i l}, M_{j}, M_{k}\right\}+(8 / 3)\left\{L_{j k}, M_{i}, M_{l}\right\}-16 \omega^{2}\left\{L_{i k}, L_{j l}\right\} \\
& +16 \omega^{2}\left\{L_{i l}, L_{j k}\right\} \text {. } \\
& \left\{Q_{i j}, R_{i j k}\right\}=(8 / 3)\left\{L_{i j}, L_{i j}, M_{k}\right\}-(8 / 3)\left\{L_{i j}, L_{i k}, M_{j}\right\} \\
& -(8 / 3)\left\{L_{i j}, L_{j k}, M_{i}\right\}-(64 / 3)\left\{L_{i j}, M_{k}\right\}-(64 / 3)\left\{L_{i k}, M_{j}\right\} \\
& -(64 / 3)\left\{L_{j k}, M_{i}\right\}+16\left(1-k_{i}^{2}\right)\left\{L_{j k}, M_{j}\right\}+16\left(1-k_{j}^{2}\right)\left\{L_{i k}, M_{i}\right\} \\
& -64\left(1-k_{i}^{2}\right)\left(1-k_{j}^{2}\right) M_{k} \\
& \left\{Q_{i j}, R_{i k l}\right\}=(8 / 3)\left\{L_{i j}, L_{i k}, M_{l}\right\}-(8 / 3)\left\{L_{i j}, L_{i l}, M_{k}\right\} \\
& -(8 / 3)\left\{L_{i k}, L_{j l}, M_{i}\right\}+(8 / 3)\left\{L_{i l}, L_{j k}, M_{i}\right\} \\
& +16\left(1-k_{i}^{2}\right)\left(\left\{L_{j l}, M_{k}\right\}-\left\{L_{j k}, M_{l}\right\}\right), \\
& \left\{Q_{i j}, R_{k l m}\right\}=-(8 / 3)\left\{L_{i k}, L_{j l}, M_{m}\right\}+(8 / 3)\left\{L_{i l}, L_{j k}, M_{m}\right\} \\
& (8 / 3)\left\{L_{i k}, L_{j m}, M_{l}\right\}-(8 / 3)\left\{L_{i m}, L_{j k}, M_{l}\right\} \\
& -(8 / 3)\left\{L_{i l}, L_{j m}, M_{k}\right\}+(8 / 3)\left\{L_{i m}, L_{j l}, M_{k}\right\} \text {. }
\end{aligned}
$$


The symmetric products of components of $R_{i j k}$ with itself are

$$
\begin{gathered}
(A .5) R_{i j k}^{2}=-(4 / 3)\left\{L_{i j}, L_{i k}, L_{j k}\right\}+(64 / 3)\left\{L_{i j}, L_{i k}\right\} \\
+(64 / 3)\left\{L_{i j}, L_{j k}\right\}+(64 / 3)\left\{L_{i k}, L_{j k}\right\}-4 L_{k k}\left\{L_{i j}, L_{i j}\right\} \\
-8\left(1-k_{j}^{2}\right)\left\{L_{i k}, L_{i k}\right\}-8\left(1-k_{i}^{2}\right)\left\{L_{j k}, L_{j k}\right\}+(128 / 3)\left(1-k_{k}^{2}\right) L_{i j} \\
+(128 / 3)\left(1-k_{j}^{2}\right) L_{i k}+(128 / 3)\left(1-k_{i}^{2}\right) L_{j k}+64\left(1-k_{i}^{2}\right)\left(1-k_{j}^{2}\right)\left(1-k_{k}^{2}\right) . \\
(A .6) \quad\left\{R_{i j k}, R_{i j l}\right\}=-(8 / 3)\left\{L_{i j}, L_{i j}, L_{k l}\right\}+(8 / 3)\left\{L_{i j}, L_{i k}, L_{j l}\right\} \\
+(8 / 3)\left\{L_{i j}, L_{i l}, L_{j k}\right\}+(64 / 3)\left\{L_{i j}, L_{k l}\right\}+(64 / 3)\left\{L_{i k}, L_{j l}\right\} \\
+(64 / 3)\left\{L_{i l}, L_{j k}\right\}-16\left(1-k_{j}^{2}\right)\left\{L_{i k}, L_{i l}\right\}-16\left(1-k_{i}^{2}\right)\left\{L_{j k}, L_{j l}\right\} \\
+64\left(1-k_{i}^{2}\right)\left(1-k_{j}^{2}\right) L_{k l}, \\
\left\{R_{i j k}, R_{i l m}\right\}=-(8 / 3)\left\{L_{i j}, L_{i l}, L_{k m}\right\}+(8 / 3)\left\{L_{i j}, L_{i k}, L_{j l}\right\} \\
+(8 / 3)\left\{L_{i k}, L_{i l}, L_{j m}\right\}-(8 / 3)\left\{L_{i k}, L_{i m}, L_{j l}\right\} \\
+16\left(1-k_{i}^{2}\right)\left(\left\{L_{j l}, L_{k m}\right\}-\left\{L_{j m}, L_{k l}\right\}\right), \\
\left\{R_{i j k}, R_{l m n}\right\}=(8 / 3)\left\{L_{i l}, L_{j m}, L_{k n}\right\}-(8 / 3)\left\{L_{i l}, L_{j n}, L_{k m}\right\} \\
-(8 / 3)\left\{L_{i m}, L_{j l}, L_{k n}\right\}+(8 / 3)\left\{L_{i m}, L_{j n}, L_{k l}\right\} \\
+(8 / 3)\left\{L_{i n}, L_{j l}, L_{k m}\right\}-(8 / 3)\left\{L_{i n}, L_{j m}, L_{k l}\right\} .
\end{gathered}
$$

Symmetric products of $R$ with $A$ are

$$
\begin{gathered}
(A .7) \quad\left\{R_{i j k}, A_{i}\right\}=\frac{8}{3}\left\{M_{i}, L_{i k}, U_{j}\right\}+\frac{8}{3}\left\{M_{k}, L_{j i}, U_{i}\right\} \\
-\frac{8}{3}\left\{M_{i}, L_{i j}, U_{k}\right\}-\frac{8}{3}\left\{M_{j}, L_{k i}, U_{i}\right\}++16\left(1-k_{i}^{2}\right)\left(\left\{M_{j}, U_{k}\right\}-\left\{M_{k}, U_{j}\right\}\right), \\
\left\{R_{i j k}, A_{\ell}\right\}=\frac{8}{3}\left\{M_{i}, L_{\ell k}, U_{j}\right\}-\frac{8}{3}\left\{M_{i}, L_{\ell j}, U_{k}\right\}+\frac{8}{3}\left\{M_{j}, L_{\ell i}, U_{k}\right\} \\
-\frac{8}{3}\left\{M_{j}, L_{\ell k}, U_{i}\right\}+\frac{8}{3}\left\{M_{k}, L_{\ell j}, U_{i}\right\}-\frac{8}{3}\left\{M_{k}, L_{\ell i}, U_{j}\right\} .
\end{gathered}
$$

Symmetric products between $R$ and $B$ are

$$
\begin{gathered}
\left\{R_{i j k}, B_{i j}\right\}=\frac{8}{3}\left\{L_{i j}, L_{j k}, U_{i}\right\}+\frac{8}{3}\left\{L_{i j}, L_{k i}, U_{j}\right\}-\frac{8}{3}\left\{L_{i j}, L_{i j}, U_{k}\right\} \\
+\frac{64}{3}\left\{L_{i j}, U_{k}\right\}+\frac{64}{3}\left\{L_{k i}, U_{j}\right\}+\frac{64}{3}\left\{L_{j k}, U_{i}\right\}-16\left(1-k_{i}^{2}\right)\left\{L_{j k}, U_{j}\right\} \\
-16\left(1-k_{j}^{2}\right)\left\{L_{k i}, U_{i}\right\}+64\left(1-k_{i}^{2}\right)\left(1-k_{j}^{2}\right) U_{k}
\end{gathered}
$$




$$
\begin{gathered}
\left\{R_{i j k}, B_{i l}\right\}=\frac{8}{3}\left\{L_{i j}, L_{k l}, U_{i}\right\}-\frac{8}{3}\left\{L_{i k}, L_{j l}, U_{i}\right\}+\frac{8}{3}\left\{L_{i k}, L_{i l}, U_{j}\right\} \\
-\frac{8}{3}\left\{L_{i j}, L_{i l}, U_{k}\right\}+16\left(1-k_{i}^{2}\right)\left(\left\{L_{j l}, U_{k}\right\}-\left\{L_{k l}, U_{j}\right\}\right), \\
\left\{R_{i j k}, B_{l m}\right\}=\frac{8}{3}\left\{L_{i l}, L_{j m}, U_{k}\right\}-\frac{8}{3}\left\{L_{i m}, L_{j l}, U_{k}\right\}+\frac{8}{3}\left\{L_{j l}, L_{k m}, U_{i}\right\} \\
-\frac{8}{3}\left\{L_{j m}, L_{k l}, U_{i}\right\}+\frac{8}{3}\left\{L_{k l}, L_{i m}, U_{j}\right\}-\frac{8}{3}\left\{L_{k m}, L_{i l}, U_{j}\right\} .
\end{gathered}
$$

The symmetric products of $A$ with itself are

$$
\begin{gathered}
\text { (A.9) } A_{i}^{2}=\frac{2}{3}\left\{M_{i}, M_{i}, L\right\}+16 \omega^{2} U_{i}^{2}+16 \omega^{2}\left(1-k_{i}^{2}\right) L-32 \omega^{2} M_{i}, \\
\left\{A_{i}, A_{j}\right\}=\frac{4}{3}\left\{M_{i}, M_{j}, L\right\}+16 \omega^{2}\left\{U_{i}, U_{j}\right\}+8 \omega^{2}\left\{L_{i j}, L\right\} .
\end{gathered}
$$

The symmetric products of $A$ and $B$ are

$$
\begin{gathered}
(A .10) \quad\left\{A_{i}, B_{i j}\right\}=\frac{8}{3}\left\{M_{i}, U_{i}, U_{j}\right\}-\frac{8}{3}\left\{M_{j}, U_{i}, U_{i}\right\}+\frac{4}{3}\left\{M_{i}, L_{i j}, L\right\} \\
+\frac{32}{3}\left\{M_{i}, M_{j}\right\}-8\left(1-k_{i}^{2}\right)\left\{M_{j}, L\right\}-\frac{64}{3} \omega^{2} L_{i j}, \\
\left\{A_{i}, B_{j k}\right\}=\frac{8}{3}\left\{M_{j}, U_{k}, U_{i}\right\}-\frac{8}{3}\left\{M_{k}, U_{j}, U_{i}\right\}+\frac{4}{3}\left\{M_{j}, L_{k i}, L\right\}-\frac{4}{3}\left\{M_{k}, L_{j i}, L\right\} .
\end{gathered}
$$

The symmetric products of $B$ with itself are

$$
\begin{gathered}
B_{i j}^{2}=\frac{8}{3}\left\{L_{i j}, U_{i}, U_{j}\right\}+\frac{2}{3}\left\{L_{i j}, L_{i j}, L\right\}+\frac{64}{3}\left\{U_{i}, U_{j}\right\}-16\left(1-k_{i}^{2}\right) U_{j}^{2} \\
-16\left(1-k_{j}^{2}\right) U_{i}^{2}+\frac{16}{3}\left\{L_{i j}, M_{i}\right\}+\frac{16}{3}\left\{L_{i j}, M_{j}\right\}-\frac{16}{3}\left\{L_{i j}, L\right\} \\
+\frac{32}{3}\left(1-k_{i}^{2}\right) M_{j}+\frac{32}{3}\left(1-k_{j}^{2}\right) M_{i}-16\left(1-k_{i}^{2}\right)\left(1-k_{j}^{2}\right) L \\
\left\{B_{i j}, B_{i k}\right\}=\frac{8}{3}\left\{L_{i j}, U_{i}, U_{j}\right\}+\frac{8}{3}\left\{L_{j k}, U_{i}, U_{j}\right\}-\frac{8}{3}\left\{L_{j k}, U_{i}, U_{i}\right\} \\
+\frac{4}{3}\left\{L_{i j}, L_{i k}, L\right\}+\frac{16}{3}\left\{L_{i j}, M_{k}\right\}+\frac{16}{3}\left\{L_{j k}, M_{i}\right\}+\frac{16}{3}\left\{L_{k i}, M_{j}\right\}- \\
\left\{B_{i j}, B_{k l}\right\}=\frac{8}{3}\left\{L_{i l}, U_{j}, U_{k}\right\}-\frac{8}{3}\left\{L_{i k}, U_{j}, U_{l}\right\}+\frac{8}{3}\left\{L_{j k}, U_{i}, U_{l}\right\} \\
-\frac{8}{3}\left\{L_{j l}, U_{i}, U_{k}\right\}+\frac{4}{3}\left\{L_{i l}, L_{j k}, L\right\}-\frac{4}{3}\left\{L_{i k}, L_{j l}, L\right\} .
\end{gathered}
$$




\section{References}

[1] E.G.Kalnins, W.Miller Jr. and G.S.Pogosyan. Superintegrability and associated polynomial solutions. Euclidean space and sphere in two-dimensions. J.Math.Phys. 37, 6439, 1996

[2] E.G.Kalnins, W.Miller Jr. and G.S.Pogosyan. Superintegrability in the threedimensional Euclidean space. J.Math.Phys., 40, 708-725, 1999.

[3] E.G.Kalnins, W.Miller Jr. and G.S.Pogosyan. Completeness of multiseparable superintegrability in $E_{2, C}$ J.Phys. A33, 4105-4120, 2000.

[4] E.G.Kalnins, W.Miller Jr. and G.S.Pogosyan. Completeness of multiseparable superintegrability on the complex 2-sphere J.Phys. A33, 6791-6806, 2000.

[5] J.Friš, Ya.A.Smorodinskii, M.Uhlír and P.Winternitz. Symmetry Groups in Classical and Quantum Mechanics; Sov.J.Nucl.Phys. 4 (1967) 444.

[6] A.A.Makarov, Ya.A.Smorodinsky, Kh.Valiev and P.Winternitz. A Systematic Search for Nonrelativistic Systems with Dynamical Symmetries; Nuovo Cimento A 52 (1967) 1061.

[7] N.W.Evans. Superintegrability in Classical Mechanics; Phys.Rev. A 41 (1990) 5666; Group Theory of the Smorodinsky-Winternitz System; J.Math.Phys. 32 (1991) 3369.

[8] W.Miller, Jr. Symmetry and Separation of Variables. Addison-Wesley Publishing Company, 1977.

[9] E.G. Kalnins and W.Miller, Jr.. Separation of variables on n-dimensional Riemannian manifolds 1 . The $n$-sphere $S_{n}$ and Euclidean $n$-space; J. Math. Phys., 27 (1986), pp. 1721-1736.

[10] E.G. Kalnins 1986 Separation of Variables for Riemannian spaces of constant curvature (Longman)

[11] C.Boyer, E.Kalnins, W.Miller, Jr. Lie Theory and Separation of Variables, 7; The Harmonic Oscillator in Elliptic Coordinates and Ince Polynomials, $J$. Math. Phys., 16, 512 (1975).

[12] W.Kallies, I.Lukach, G.S.Pogosyan and A.N.Sissakian. Ellipsoidal Bases for Harmonic Oscillator; Proceedings of the "International Workshop on 'Symmetry Methods in Physics' in Memory of Prof. Ya. A. Smorodinsky”, Vol.1, 
206, Publishing Department of JINR, Dubna (1994) Preprint JINR, E2-94230, Dubna, 1994.

[13] Ye.M.Hakobyan, G.S.Pogosyan and A.N.Sissakian. On a Generalized Ddimensional Oscillator. Interbasis Expansions, Physics of Atomic Nuclei (Yadernaya Fizika), 61 (10), 1762-1767, 1998.

[14] E.G. Kalnins and W. Miller, Jr. Killing tensors and variable separation for Hamilton-Jacobi and Helmholtz equations. SIAM J. Math. Anal. 11, 10111026, (1980).

[15] A.J.Macfarline, Nucl. Phys., B386, 453, 1992.

[16] C.P.Boyer, E.G.Kalnins and W.Miller Jr. Stäckel - equivalent integrable Hamiltonian systems SIAM J. Math. Anal., 17, 778-797, 1986.

[17] E.Kalnins and W.Miller, Jr. Separable coordinates, integrability and the Niven equations. J.Phys., A25, 5663-5675, 1992. 